\title{
Variations on cosmology and astrophysics
}

\section{Joseph Silk}

The Physics-Astronomy Frontier. By Fred Hoyle and Jayant Narlikar. Pp.483. ISBN 0-7167-1160-5. (W.H. Freeman: 1981.) $£ 16.60, \$ 23.95$. Relativistic Cosmology: An Introduction. By Jean Heidmann. Pp.168. ISBN 0-387-10138-1. (SpringerVerlag: 1980.) DM48, \$28.30. Cosmology, 2nd Edn. By Michael Rowan-Robinson. Pp.152. Hbk ISBN 0-19-851857-9; pbk ISBN 0-19-851858-9. (Oxford University Press/Clarendon: 1981.) Hbk £12.50, $\$ 44.50$; pbk £5.95, $\$ 17.95$.

IN ANY rapidly developing area of science, the textbook writer should do more than prepare a guide, no matter how thorough, to past accomplishments in his field. He must capture some of the excitement of developments in research, of new and tentative discoveries, of competing claims and counterclaims. An up-to-date treatment is especially crucial in the field of astrophysics, where the student is confronted in the daily press with a barrage of half-digested reports of new advances.

The texts by Jean Heidmann and by Fred Hoyle and Jayant Narlikar are failures by this measure. This defect is unfortunate since both books possess refreshingly novel features. Heidmann's text is a translation from French, originally published in 1973. Few revisions were made for the English edition, affecting only about five per cent of the text. The field of cosmology has developed enormously in the past decade; little of this is reflected in Relativistic Cosmology. However an unusual feature is the attempt, on the whole successful, to make esoteric topics in differential geometry accessible to the non-specialist.

The book begins with a discussion of topics in extragalactic astronomy that are of interest to the aspiring cosmologist, including clustering of galaxies, the expansion of the Universe, and intergalactic matter. The text, though, suffers from a lack of modern results. Although the intent is for the reader to fill in details by reference to the bibliographies, this will be difficult, since the references, almost without exception, stop at 1978. The section on the differential geometry of curved spaces is lucid and interesting. My only quibble is that it is almost irrelevant to the rest of the book. The remaining sections consist of a discussion of the Friedmann-Lemaitre cosmological models and of rudimentary observational cosmology. While the level of this book is appropriate for a first-year course for an undergraduate with a mathematics and physics background, it is difficult to imagine any course being based on this text without an enormous amount of supplementary material.

By way of contrast, The PhysicsAstronomy Frontier does provide a selfcontained account of astrophysics. Its aim is to describe astronomy from the perspective of a physicist. Consequently, each section begins with a discussion of one of magnetic, nuclear weak and strong, and gravitational) before moving on to the astronomical phenomena. This book is especially interesting on historical developments and anecdotes - such as when Hoyle reminisces about how Walter Baade almost discovered the pulsar in the Crab nebula - and the early years of radio and $\mathrm{X}$-ray astronomy are well covered. However, the authors do not mention any observational discoveries of the past five years. Consequently, the explosive growth in radio and $\mathrm{X}$-ray astronomy and other fields is ignored. This is especially unfortunate in a book that purports to be probing the frontiers of astronomy and physics. One notable omission is any discussion of particle physics in the very early stages of the big bang. Instead, the reader is offered an exotic cosmological theory, developed, and possibly only taken seriously, by the authors themselves.

The Physics-Astronomy Frontier is written for students who possess a strong secondary school science background. The text is mostly non-mathematical. Does it succeed, as the authors hope, by focusing the fundamental forces of nature (electro-

on the fundamental forces of nature in enabling the reader to actually acquire a physical understanding of astronomical phenomena? Certainly it is far superior in this regard to other astronomy texts at a comparable level. However, the astronomical contents are deficient and dated; at best, one might use the Hoyle-Narlikar book to complement a more conventional astronomy text assigned for an undergraduate course for non-specialists.

Michael Rowan-Robinson's second edition of Cosmology is an updated version of a text originally published in 1975 for first-year mathematics and physics undergraduates. It provides a selfcontained description of the visible Universe, including both galactic and extragalactic phenomena of interest to the observational cosmologist. There are minor omissions, such as any discussion of chemical evolution, but a reasonably thorough basis is established for cosmological theory. The meat of the book lies in its discussion of the big bang models and of their observational implications. This is a well-written summary of cosmology, which aspires (not without good reason) to be a successor to Hermann Bondi's text of the same title that had such a profound influence on undergraduates two or three decades ago.

Joseph Silk is Professor of Astronomy at the University of California, Berkeley.

\section{Life of cosmic rays}

\section{G.T. Bath}

High Energy Astrophysics. By M.S. Longair. Pp.412. Hbk ISBN 0-521-23513-8; pbk ISBN 0-521-28013-3. (Cambridge University Press: 1982.) Hbk $£ 24, \$ 49.95$; pbk $£ 8.95, \$ 19.95$.

IT Is a considerable task of synthesis to show the connections between the main fields of astronomy which have flourished in recent years and at the same time convey the drama of research at the frontiers. It must range from the solar wind to clusters of galaxies, from radiation processes in the presence of strong magnetic fields to the space-time structure of black holes. This is the ambitious aim of this introductory textbook - to show how astronomers work, how they think and how the subject has developed - by introducing the reader to many of the discoveries of the past 20 years, and by explaining some of the theoretical interpretation that has survived the rigours of criticism and discussion.

But this book is not simply an exposition of modern astrophysics. The author adopts a quite original approach. Taking as his central theme the subject of cosmic rays, their detection and the physics of their interaction with the environment, the presentation derives the necessary basic physics to trace the cosmic ray propagation path through the atmosphere, the magnetosphere, the solar wind, and the interstellar and intergalactic medium, back to the production of high energy particles in galactic and extra-galactic objects. The high energy astrophysics of the title refers to these ultimate sources of cosmic rays in the Universe, which are introduced as their peregrinations through the hazards of space are traced back to their birth.

The author makes no apology for presenting the material, and referring to it, as a lecture course. Indeed his personality and enthusiasm shine through the informal and stimulating style of the writing. The depth of discussion varies from subject to subject. In a book which introduces cosmic ray physics and detectors, basic astronomy at all wavelengths, stellar evolution and stellar death, the properties of galaxies and clusters of galaxies, and high energy astrophysics, this is not surprising. As a stimulating undergraduate introduction to particle propagation at high energies, and the astrophysical environment in which it all takes place, this book gives a broad background without losing contact with the dynamism that created the subject.

G.T. Bath is a Research Fellow in the Department of Astrophysics, University of Oxford. 